\title{
An epigenetic target for synthetic lethality
}

Genome-wide sequencing studies have revealed that the gene ARIDIA, which encodes a component of a chromatin remodelling complex, is among the most frequently mutated genes in human cancers. Now, reporting in Nature Medicine, Zhang and colleagues show that inactivation or absence of ARID1A acts in a synthetic lethal manner with the inhibition of the methyltransferase EZH2 - opening up new therapeutic possibilities for cancers with ARID1A mutations.

The highest mutation rate for ARID1A had been reported in ovarian clear cell carcinomas (OCCCs), with approximately half of the samples being found to carry frame-shift or nonsense mutations that lead to the functional loss of ARID1A.

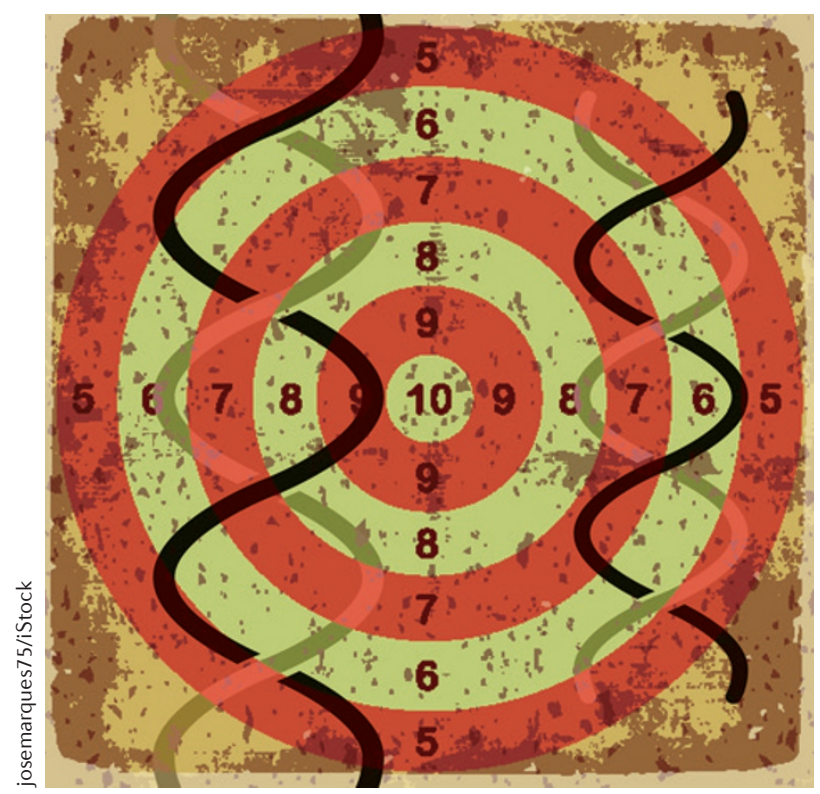

As ARID1A is involved in the epigenetic regulation of genes

Intraperitoneal

injection of

GSK126

caused marked

tumour

regression in

two different

orthotopic

xenograft

models

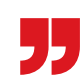
the authors tested whether its (it is a member of the SWI-SNF

nucleosome remodelling complex), deficiency might render cells vulnerable to the inhibition of a second epigenetic regulator. To test this, $3 \mathrm{D}$ cultures of OCCC cell lines with wild-type ARID1A were established, and ARID1A was knocked down by short interfering RNA (siRNA). Then, 15 commercially available small molecule inhibitors of epigenetic regulators were tested for their ability to kill ARID1A-deficient cells but not their ARID1A wild-type counterparts.

The compound with the highest selectivity for ARID1A-deficient cells was GSK126, a highly specific inhibitor of $\mathrm{EZH} 2$, which is a histone methyltransferase that is part of the polycomb repressive complex 2 . EZH2 catalyses lysine 27 trimethylation on histone $\mathrm{H} 3$ (H3K27Me3), thereby silencing gene expression.

Interestingly, the polycomb complex and the SWI-SNF complex are known to have antagonistic effects on the expression of a number of genes. To elucidate the underpinnings of the synthetic lethal relationship between ARID1A and EZH2, the authors searched for genes that are differentially regulated by the two proteins. They found a significant overlap of genes that are upregulated by either ARID1A restoration or by EZH2 inhibition. The most interesting of these genes was PIK3IP1 (phosphoinositide 3-kinase interacting protein 1 ), encoding a negative regulator of the phosphoinositide 3-kinase (PI3K)-AKT pathway, which is a well-known oncogenic signalling axis.

ARID1A and EZH2 were found to associate with the promoter of PIK3IP1. Based on further biochemical experiments, the authors propose that ARID1A, which activates PIK3IP1 expression, usually dominates over EZH2, which suppresses PIK3IP1 expression. When ARID1A is absent, EZH2 silences PIK3IP1. Subsequent inhibition of $\mathrm{EZH} 2$, in turn, reinstates the expression of PIK3IP1 - and thereby the inhibition of the PI3K-AKT pathway.

To test whether this relationship can be exploited in vivo, ARID1A-mutated OCCC cells were used to establish tumours in mice. Intraperitoneal injection of GSK126 caused marked tumour regression in two different orthotopic xenograft models, and reduced metastases in a model of disseminated OCCC. Immunohistochemical staining showed lower levels of activity of the PI3K-AKT pathway in tumours of GSK126-treated mice than in vehicle-treated tumours.

These results point to a novel synthetic lethal strategy based on inhibition of EZH2 in ARID1Amutated cancers. Given the frequency of ARID1A mutations, this approach may potentially have wide-ranging applications.

Alexandra Flemming

ORIGINAL RESEARCH PAPER Bitler, B. G. et al. Synthetic lethality by targeting $\mathrm{EZH} 2$ methyltransferase activity in ARID1A-mutated cancers. Nature Med. http://dx.doi.org/10.1038/nm.3799 (2015) 